\title{
Incidence and etiology of infectious diarrhea from a facility-based surveillance system in Guatemala, 2008-2012
}

\author{
Wences Arvelo ${ }^{1 *}$, Aron J. Hall², Olga Henao ${ }^{3}$, Beatriz Lopez ${ }^{4}$, Chris Bernart ${ }^{4}$, Juan C. Moir ${ }^{5}$, Lissette Reyes ${ }^{5}$, \\ Susan P. Montgomery', Oliver Morgan ${ }^{3}$, Alejandra Estevez ${ }^{4}$, Michele B. Parsons $^{3}$, Maria R. Lopez ${ }^{4}$, Gerry Gomez $^{3}$, \\ Jan Vinje ${ }^{2}$, Nicole Gregoricus' ${ }^{2}$ Umesh Parashar², Eric D. Mintz ${ }^{3}$, John McCracken ${ }^{4}$, Joe P. Bryan ${ }^{1}$ and \\ Kim A. Lindblade ${ }^{1}$
}

\begin{abstract}
Background: Diarrhea is a major cause of morbidity and mortality, yet incidence and etiology data are limited. We conducted laboratory-based diarrhea surveillance in Guatemala.

Methods: A diarrhea case was defined as $\geq 3$ loose stools in a 24-h period in a person presenting to the surveillance facilities. Epidemiologic data and stool specimens were collected. Specimens were tested for bacterial, parasitic, and viral pathogens. Yearly incidence was adjusted for healthcare seeking behaviors determined from a household survey conducted in the surveillance catchment area.

Results: From November 2008 to December 2012, the surveillance system captured 5331 diarrhea cases; among these 1381 (26\%) had specimens tested for all enteric pathogens of interest. The adjusted incidence averaged 659 diarrhea cases per 10,000 persons per year, and was highest among children aged $<5$ years, averaging 1584 cases per 10,000 children per year. Among 1381 (26\%) specimens tested for all the pathogens of interest, 235 (17\%) had a viral etiology, 275 (20\%) had a bacterial, 50 (4\%) had parasites, and 86 (6\%) had co-infections. Among 827 (60\%) specimens from children aged < 5 years, a virus was identified in 196 (23\%) patients; 165 (20\%) had norovirus and 99 (12\%) rotavirus, including co-infections. Among 554 patients aged $\geq 5$ years, 103 (19\%) had a bacterial etiology, including diarrheagenic Escherichia coli in 94 (17\%) cases, Shigella spp. in 31 (6\%), Campylobacter spp. in 5 (1\%), and Salmonella spp. in 4 (1\%) cases. Detection of Giardia and Cryptosporidium was infrequent (73 cases; 5\%).

Conclusions: There was a substantial burden of viral and bacterial diarrheal diseases in Guatemala, highlighting the importance of strengthening laboratory capacity for rapid detection and control and for evaluation of public health interventions.
\end{abstract}

\section{Background}

Diarrheal diseases are a major cause of morbidity and mortality worldwide, particularly among children and the elderly [1-3]. In developing countries, diarrhea is the second leading cause of mortality in children $<5$ years of age [4]. Annually, approximately four billion diarrhea episodes and 1.3 million diarrhea-related deaths occur worldwide [1]. Despite the magnitude of diarrheal diseases, these are only

\footnotetext{
* Correspondence: warvelo@cdc.gov

${ }^{1}$ Center for Global Health, US Centers for Disease Control and Prevention,

1600 Clifton Road, NE, Mailstop V24-5, Atlanta, GA 30333, USA

Full list of author information is available at the end of the article
}

estimates derived from different data sources gathered through varying methodologies $[1,4-6]$. These estimates have limitations that may lead to underreporting and an underestimation of the actual burden. For example, healthcare workers might face challenges for consistently reporting diarrheal episodes. Milder illnesses often never reach a reporting facility. Lastly, healthcare utilization practices are complex, and social, economic, geographic, and individual factors affect healthcare seeking behaviors $[7,8]$.

The US Centers for Disease Control and Prevention (CDC) in collaboration with the Ministerio de Salud Publico y Asistencia Social and the Universidad del Valle

(c) The Author(s). 2019 Open Access This article is distributed under the terms of the Creative Commons Attribution 4.0 International License (http://creativecommons.org/licenses/by/4.0/), which permits unrestricted use, distribution, and 
de Guatemala (UVG), initiated an active laboratorybased surveillance system for diarrheal, respiratory, febrile illness, and acute infectious neurological diseases in two departments in Guatemala. Coupled with laboratory testing, the main objectives of this surveillance system were to determine the etiology-specific burden of these syndromes and serve as a platform for evaluating the effectiveness or impact of interventions such as, vaccines, zinc, and campaigns for use of oral rehydration therapy. Given the limited information on healthcare utilization practices in Guatemala, before launching the surveillance system we also conducted household surveys to understand healthcare utilization practices and adjust disease incidence. In this report we describe healthcare utilization practices for diarrhea in two sites in Guatemala, estimate the incidence of diarrheal disease, and describe the predominant bacterial, viral, and parasitic pathogens detected. More comprehensive pathogen specific clinical characterization and burden of illness has been published elsewhere.

\section{Methods}

\section{Sites}

Guatemala is divided into 22 departments which are in turn divided into municipalities. The household surveys and the surveillance system were conducted in the departments of Santa Rosa and Quetzaltenango (Fig. 1). The sites were purposely selected based on logistical and political factors. In the 2002 national census, the most recently available census at the time of the study, the department of Santa Rosa had a population of 300,928, and Quetzaltenango had a population of 623,494 persons.

Government healthcare facilities in these departments included hospitals, and ambulatory centers. Cuilapa Regional Hospital is a 176-bed referral hospital in Santa Rosa, and serves Santa Rosa and the neighboring departments of Jutiapa and Jalapa. The Hospital del Occidente (Western Regional Hospital) in Quetzaltenango is a 425-bed hospital, and also serves the neighboring departments of Huehuetenango, San Marcos, Totonicapán. Other sources of healthcare in these departments include smaller public hospitals, private clinics, private hospitals, pharmacies, and traditional healers.

Before initiating surveillance, we conducted household surveys to describe healthcare utilization practices and treatment for diarrheal episodes. In Guatemala, the National Census Bureau defines an enumeration area, as the discrete geographical area for counting individuals. In the Department of Santa Rosa, we stratified enumeration areas obtained from the Guatemala National Census Bureau by those with immediate access to a hospital or health centers and those without immediate access to these health facilities. Selection of enumeration areas was conducted by probability proportional to population size based on the 2002 census data. Thirty enumeration areas were selected per stratum for a total of 60 enumeration areas. Sketch maps denoting the general layout of main structures within each enumeration area were obtained, and 20 structures were randomly selected. Once the field teams reached a selected structure they attempted to interview all persons living in the household. A household was defined as an individual or group of individuals who lived in that residential structure, including sleeping most of the nights and eating most of their meals, for at least 6 months in the 12 months prior to interview. If a structure was determined to be abandoned or non-residential, it was replaced with the next nearest until 20 households in each enumeration area were enrolled.

In Quetzaltenango the study population included persons living in municipalities served by Western Regional Hospital, which based on hospital records included the capital city and its nine surrounding rural municipalities of Almolonga, Cantel, Concepción Chiquirichapa, La Esperanza, Olintepeque, Salcajá, San Juán Ostuncalco, San Mateo and Zunil. We selected two representative samples: one from the higher density urban area in the capital, and another from the surrounding rural and semi-urban areas. In the urban area we took a stratified simple random sample of households using geospatial sampling. The district was first stratified into 26 strata based on administrative areas. We used proportional allocation (self-weighting) to determine the number of households required in each stratum by dividing the population of each stratum by the total population of the district and multiplying by 710 , the number of required households based on sample size calculations. Within each stratum we conducted a simple random selection of households without replacement using geospatial sampling, by taking a random selection of coordinates in each stratum and selecting the nearest household to that coordinate. To achieve this, we first demarcated boundaries for each urban stratum using an engineering map in AutoCAD software provided by the municipal planning department. We then plotted the boundary coordinates onto high-resolution aerial photographs available from the Guatemalan Instituto Geográfico Nacional (National Geographic Institute) that had been overlaid onto a topographic map. The aerial images were taken in 2006. Within each demarcated stratum we randomly selected coordinates, which we refer to as sample points. We then plotted the sample points onto the high-resolution aerial photographs. We then selected the nearest residential structure within a $100 \mathrm{~m}$ radius from the sample point. If two or more residential structures were a similar distance from the sample point, we used a random number table to select one of them. If there were two or more households in the selected 


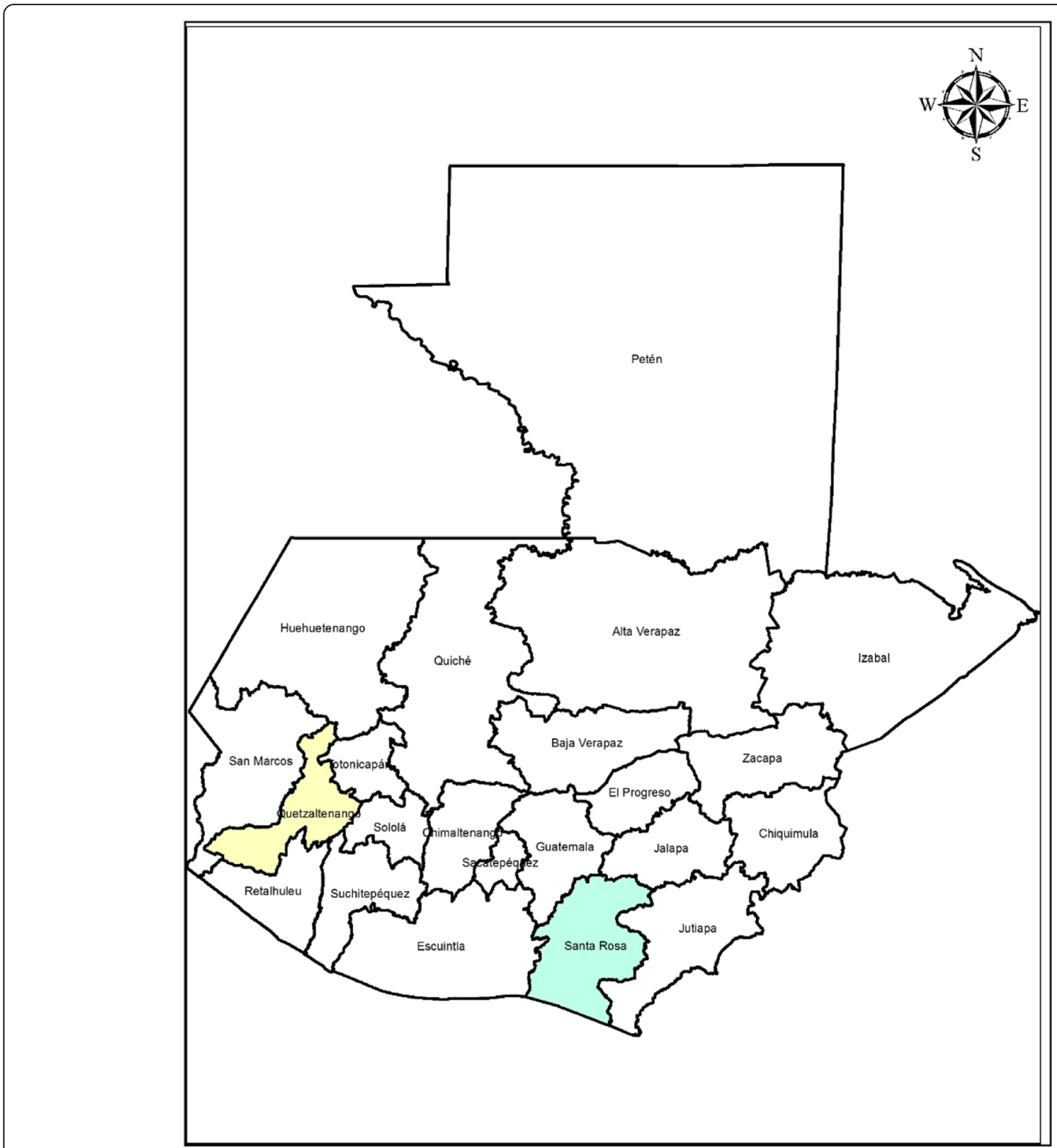

Fig. 1 Map of Guatemala highlighting Santa Rosa and Quetzaltenango, the two departments where the healthcare seeking practices surveys and the surveillance system for diarrheal diseases were implemented

residential structure, we used a simple random number table to select one of them. If no residential structure was within $100 \mathrm{~m}$ of the sample point, the sample point was replaced. For rural areas we took a two-stage cluster sample where the first stage was a sample of communities and the second stage was a sample of households within each selected community. For the first sampling stage we selected 35 clusters of 39 households to achieve the desired sample size of 1365 households. We selected clusters using the PROC SURVEYSELECT command in Statistical Analysis System version 9.3 ([SAS], SAS Institute, Cary, NC). For the second sampling stage we took a simple random sample of households within each cluster using geospatial sampling. This process was similar to the one described above for the urban area except that, for demarcation of communities, we visited each community and with a local guide demarcated the communities' boundaries using a handheld global positioning system (GPS) device.

For both surveys, interviews were conducted in person using a standardized questionnaire, administered in the local language by trained interviewers. The survey asked about episodes of diarrhea during the last 30 days before the interview for all members of the household. Question were asked to characterize the diarrheal episode, such as number of episodes in $24 \mathrm{~h}$, blood in stools, 
fever, etc. We also asked about healthcare seeking practices, and whether they visited a hospital, ambulatory clinic, private physician, pharmacy, traditional healer, among others. In addition, we asked about treatments during that episode of diarrhea, including antibiotics, rehydration solutions, herbal remedies, etc. The survey also collected information on demographic and socioeconomic characteristics (Additional file 1). All persons who had lived in the house for 6 months or more in the 12 months before the interview were eligible. Infants $<6$ months of age were included if they lived in the household since birth. For anyone who died $<30$ days before the interview, young children, or those absent at the time of the interview, questions were asked of a surrogate household member whether the deceased had experienced diarrhea in the 30 days prior to dying. In Santa Rosa, data were collected on paper forms and scanned into a Microsoft Access 2003 (Microsoft Corporation, Seattle, WA) database using TeleForms (Hewlett Packard Enterprise, Palo Alto, CA). In Quetzaltenango, the survey was conducted using personal digital assistant devices (PDAs).

\section{Surveillance}

In Santa Rosa (Fig. 2a) the surveillance system initiated in July 2007 at Cuilapa Regional Hospital and the six health centers (Nueva Santa Rosa, Cacalotepeque, Chapas, Estanzuelas, Jumaytepeque, and Ojo de Agua- Fig. 2b) in the municipality of Nueva Santa Rosa. In Quetzaltenango surveillance began in February 2009 at the Western
Regional Hospital and the four surrounding health centers in Cantel, Concepcion Chiquirichapa, La Esperanza, and Xecam (Fig. 3a). Surveillance systems in both sites are ongoing.

At the hospitals, trained nurses identified patients admitted with signs or symptoms suggestive of diarrhea by reviewing ward registers for diarrhea-related admission diagnoses or chief complaints emergency department registries. At ambulatory facilities, nurses screened patients presenting for treatment for diarrhea-related symptoms. A case of acute diarrhea was defined as $\geq 3$ loose stools in a 24-h period during the previous 7 days in a person of any age admitted to the hospital or presenting to the health centers. Patients with diarrhea $>7$ days were excluded. Clinical and epidemiologic data were collected using standardized questionnaires (Additional file 2).

We attempted to collect whole stool specimens from enrolled patients. When whole stool was not obtainable for children $<5$ years old, we collected a rectal swab [9]. Specimens were kept at $4{ }^{\circ} \mathrm{C}$ and transported within $24 \mathrm{~h}$ to the laboratory. Specimens were tested for enteric viruses, bacteria, and parasites [7, 10, 11]. Rotavirus testing was done via qualitative enzyme immunoassay (EIA) for the detection of Group A rotavirus with the IDEIA Rotavirus test (Thermo Fisher Scientific Inc., Waltham, MA) [7]. Norovirus testing for genogroup I and II was done by real-time reverse transcription quantitative polymerase chain reaction (RT-qPCR) [12]. We tested for enteric bacteria including Campylobacter, Salmonella, Shigella,
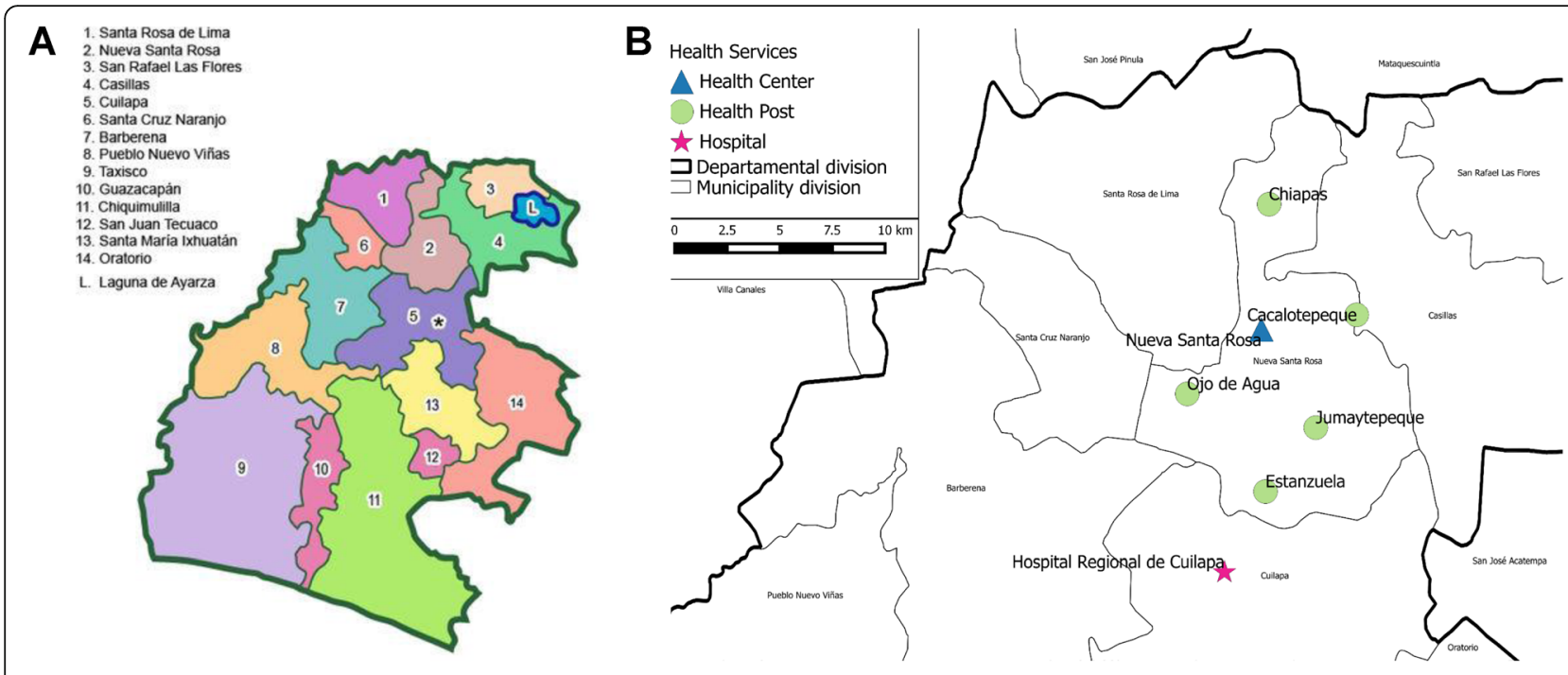

Fig. 2 a: Political map showing municipalities in the Department of Santa Rosa. The Health Utilization Survey was conducted across the entire Department of Santa Rosa. The municipality of Nueva Santa Rosa is number 2 on the map at left and the capital of Cuilapa where the regional hospital is located is marked with a star. Surveillance for disease at the hospital includes all municipalities except the coastal municipalities of Taxisco, Guazacapan and Chiquimulilla as residents of these municipalities are more likely to go to a neighboring department (Esquintla) because of transportation access. b: Municipality of Nueva Santa Rosa where disease surveillance is conducted including the town of Nueva Santa Rosa where the health center is located and the health posts in Cacalotepeque, Chapas, Estanzuelas, Jumaytepeque, and Ojo de Agua 


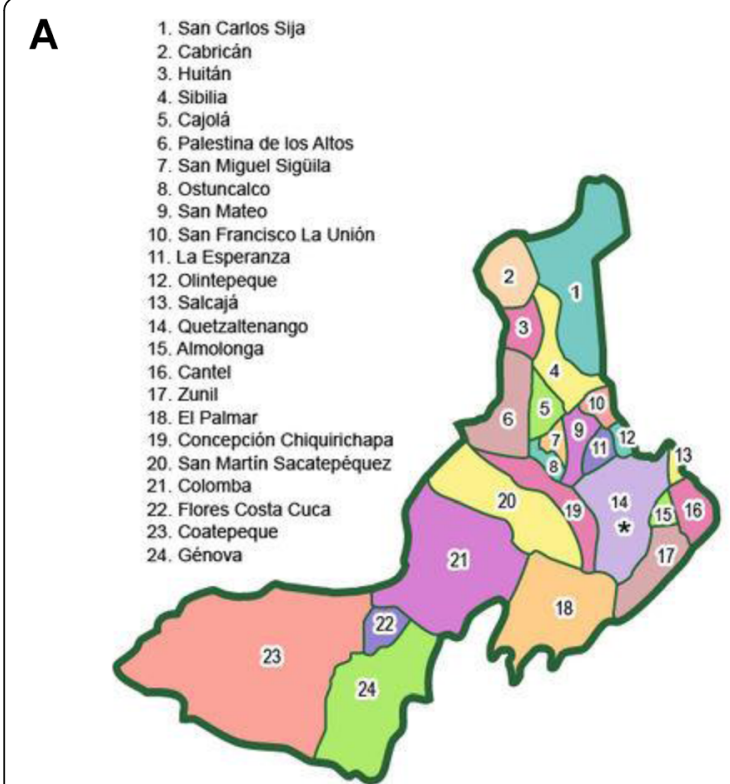

B

Fig. 3 a: Political map showing municipalities in the Department of Quetzaltenango. Quetzaltenango, the capital and the location of the regional hospital and its nine surrounding rural municipalities of Almolonga (15), Cantel (16), Concepción Chiquirichapa (19), La Esperanza (11),

Olintepeque (12), Salcajá (13), San Juán Ostuncalco (8), San Mateo (9) and Zunil (17) are the locations of the health utilization survey. Disease surveillance was conducted at the Western Regional Hospital capturing hospitalized cases from across the department of Quetzaltenango, and in four surrounding ambulatory facilities, namely the health centers in Cantel, Concepcion Chiquirichapa, La Esperanza, and Xecam. b: Health Centers in Concepcion Chiqurichapa, La Esperanza, Cantel and Xecam and Hospital Regional de Occidente where disease surveillance was conducted in Quetzaltenango Department

and diarrheagenic Escherichia coli ${ }^{12}$. Salmonella and Shigella were cultured using routine bacteriological methods, while for Campylobacter we used selective agar base, Karmali (Oxoid, Basingstoke, UK) and incubated at $42^{\circ} \mathrm{C}$ for $48 \mathrm{~h}$ under microaerophilic conditions provided by the CampyGen ${ }^{\text {ma }}$ generating system (Oxoid, Basingstoke, UK) $)^{13}$. E. coli testing was performed on bulk stool specimens by conventional PCR to detect the presence of specific virulence determinants of enterotoxigenic $E$. coli, enteropathogenic E. coli and shiga-toxin producing E. coli. ${ }^{14,15}$. Examination for protozoa and soil transmitted helminths was conducted microscopically by direct smear examination. Cryptosporidium spp. and Giardia were also detected by direct fluorescent antibodies (http://www.meridianbioscience.com/diagnostic-products/cryptosporidium-and-giardia/merifluor/merifluorcryptosporidium-and-giardia.aspx $)^{9}$.

\section{Data management and analysis}

Data were collected in the field by trained staff using PDAs. Data were managed and stored using Microsoft SQL Server (Microsoft Corporation, Seattle, WA). All data are kept in secured servers at the UVG given they contain personally identifiable and sensitive clinical information protected by the Health Insurance Portability and Accounting Act (HIPAA). Redacted deidentified versions of the data are available upon request.
We analyzed data using SAS version 9.1 (SAS Institute, Cary, NC). From the household surveys we calculated the proportion of patients seeking health-care as the number of participants who reported diarrhea and the proportion who sought care at a government health facility by age group, with $95 \%$ confidence intervals (CI) taking into account the survey design. For incidence calculations we used the cases detected by the surveillance system, including from hospitals and ambulatory sites. Yearly incidences were calculated by dividing the total number of diarrhea cases (hospitalized and ambulatory) by the mid-year population. Adjusted rates were calculated by dividing crude rates by the proportion of patients with diarrhea who reported seeking health care at a government facility in the household surveys. Each mid-year population was estimated from the most recently available 2002 national census data, as reported by the Guatemalan National Institute for Statistics.

We also conducted an etiologic analysis limited to those diarrhea cases captured from July 2008 to July 2009 for which testing was done for all the bacterial, parasitic, and viral enteric pathogens under surveillance. We defined a bacterial etiology as a case with Campylobacter spp., diarrheagenic E. coli (including enterotoxigenic, enteropathogenic, and Shiga-toxin producing $E$. coli), Salmonella spp., or Shigella spp.; a viral etiology as a case with confirmed rotavirus or norovirus; and a 
parasitic etiology as one with Giardia lamblia (also known as Giardia intestinalis or Giardia duodenalis) or Cryptosporidium spp. The etiologic analysis was also stratified by age group and by type of facility (hospital and ambulatory).

\section{Ethics approval and consent to participate}

Protocols for both the household surveys and the surveillance system received approval from the institutional review boards of the US Centers for Disease Control and Prevention (Atlanta, GA; CDC IRB protocol \#5150), and of the Universidad del Valle de Guatemala (Guatemala City, Guatemala). In addition, the protocol was reviewed and received approval from the Guatemalan Ministry of Public Health and Welfare (Guatemala City, Guatemala). The protocols included institutional review board approval to enroll minors $<17$ years of age with consent from the parent or caregiver, and assent if the child was 7 years of age or older.

For the household surveys, heads of household $\geq 18$ years old were asked for written, informed consent for them and the members of the household to participate in the survey. Head of household was defined as the primary caregiver of most children in household or the oldest person $>18$ years old in the household. All other members of the household $\geq 18$ years old were also asked to provide verbal consent, and children aged 7 through 17 years provided verbal assent, as approved by institutional review boards. Verbal consent was approved as there was no risk for the household members to participate in a small subset of question from the survey, and heads of households had provided written informed consent.

For the surveillance system, patients $\geq 18$ years old and caregivers of children $<18$ years old had to provide written, informed consent in order for them or their children to participate in the surveillance. For children aged 7 through 17 years, written assent was required in addition the caregivers written consent. For children younger than seven years we only obtained written consent to participate from their caregivers, as approved by the institutional review boards. Trained nurses informed participants about the study, including that their participation was voluntary and confidential. An informational sheet with a description of the study and contact information for the investigators was also provided. Signed consent forms were delivered to the investigators at the UVG daily.

\section{Results}

\section{Household surveys}

In Santa Rosa we enrolled 5449 persons in 1131 households from October 10 through December 13, 2006. Diarrhea in the last 30 days was reported by 375 [7\%; 95\% CI: 6-8\%] persons. Among those who reported diarrhea, 184 (49\%; 95\% CI: 44-54\%) were female and 90 (24\%; 95\% CI: 20-29\%) were children < 5 years old. In terms of healthcare seeking practices, 228 (61\%; 95\% CI: 56-66\%) persons sought care for their diarrheal illness outside their home, and 87 (23\%; 95\% CI: 19-28\%) visited a government healthcare facility (Table 1). In Quetzaltenango we enrolled 9668 persons in 1851 households from March 3 through June 11, 2009. Diarrhea in the last 30 days was reported by 747 (8\%; $95 \%$ CI: 7-8\%) persons. Among those who reported diarrhea, 380 (51\%; 95\% CI: 47-54\%) were female and 167 (19\%; 95\% CI: $15-22 \%)$ were children $<5$ years old. In terms of healthcare seeking practices, 480 (65\%; 95\% CI: 61-69\%) persons sought care for their diarrheal illness outside the home, and 360 (48\%; 95\% CI: 45-52\%) visited a government healthcare facility (Table 1 ).

\section{Surveillance}

From November 2008 to December 2012, there were 5331 case detected by the surveillance system; 3947 cases were from patients residing in the Department of Santa Rosa and 1384 from the Department of Quetzaltenango. The incidence of diarrhea among persons attending the government health care facilities from the catchment areas was 134/10,000 patients/year. However,

Table 1 Healthcare seeking practices among persons who reported having diarrhea during a community household survey in Santa Rosa and Quetzaltenango, Guatemala

\begin{tabular}{|c|c|c|c|c|c|c|}
\hline \multirow{3}{*}{$\begin{array}{l}\text { Age } \\
\text { group } \\
\text { (years) }\end{array}$} & \multicolumn{3}{|c|}{ Santa Rosa } & \multicolumn{3}{|c|}{ Quetzaltenango } \\
\hline & \multirow{2}{*}{$\begin{array}{l}\text { Reported } \\
\text { Diarrhea } \\
\text { N }\end{array}$} & \multicolumn{2}{|c|}{ Sought Care at Surveillance Facility } & \multirow{2}{*}{$\begin{array}{l}\text { Reported } \\
\text { Diarrhea } \\
\text { N }\end{array}$} & \multicolumn{2}{|c|}{ Sought Care at Surveillance Facility } \\
\hline & & $n$ & $(\%, 95 \mathrm{Cl})$ & & $n$ & $(\%, 95 \mathrm{Cl})$ \\
\hline$<1$ & 13 & 6 & $(46,23-71)$ & 22 & 9 & $(41,23-61)$ \\
\hline $1-4$ & 77 & 29 & $(38,28-49)$ & 146 & 35 & $(24,18-32)$ \\
\hline $5-19$ & 111 & 21 & $(19,13-27)$ & 213 & 22 & $(10,7-15)$ \\
\hline $20-49$ & 86 & 17 & $(20,13-29)$ & 237 & 29 & $(12,9-17)$ \\
\hline$>50$ & 88 & 14 & $(16,10-25)$ & 129 & 11 & $(9,5-15)$ \\
\hline Total & 375 & 87 & $(23,19-28)$ & 747 & 106 & $(14,12-17)$ \\
\hline
\end{tabular}


the average estimated community incidence across both sites adjusted for health care utilization was 659 diarrhea cases per 10,000/persons per year (range: 587-783 diarrhea cases per 10,000 persons per year). The adjusted incidence was highest among children $<5$ years old, averaging 1584 cases per 10,000 children per year (range: 1401-1767 cases per 10,000 children per year) (Fig. 4).

During 2008-2009 surveillance period, there were 1381 (26\%) diarrhea cases enrolled that had specimens tested for all the pathogens of interest. Overall, 235 (17\%) had only viral pathogens, 275 (20\%) had only bacterial pathogens, and $50(4 \%)$ had only parasites (Table 2). Eighty-six (6\%) cases had co-infections across etiological. Among 827 (60\%) specimens from children $<5$ years old, a viral etiology was identified in 196 (23\%): norovirus was detected in $165(20 \%)$ and rotavirus in 99 $(12 \%)$ cases (Table 3$)$. In contrast, bacterial etiologies were the most frequently identified etiology for all age groups except among infants $<1$ years old (Table 2). Excluding infants, among the 1007 patients of all other age groups, $227(23 \%)$ had a bacterial etiology. Among patients aged $\geq 5$ years, diarrheagenic Escherichia coli was detected in 94 (17\%) cases, Shigella spp. in $30(6 \%)$, Campylobacter spp. in 5 (1\%), and Salmonella spp. in 4 (1\%) cases (Table 3). Identification of protozoa (Giardia or Cryptosporidium) was infrequent with 73 cases overall. Protozoa were detected predominantly in the ambulatory setting where 63 (5\%) patients had Giardia and 9 (1\%) had Cryptosporidium. Among those aged 5-19 years, 9\% had Giardia or Cryptosporidia. Overall 6\% of patients had multiple pathogens detected, including $10 \%$ in those $<1$ year old.

\section{Discussion}

We report population-based incidence estimates for diarrheal diseases across two sites in Guatemala. The highest incidence adjusted for healthcare seeking behavior was among children $<5$ years of age with 1584 cases per 10,000 children per year. The etiologic profile of pathogens detected were similar to findings from a multinational study where rotavirus, Cryptosporidium, Shigella and pathogenic E. coli were significantly associated with moderate to severe diarrhea in children [13]. In our study, among children $<1$ year old the main etiologies were viral. Norovirus was found in a third of hospitalized children, and 17\% from ambulatory clinics. Rotavirus accounted for $23 \%$ of hospitalizations and $8 \%$ of ambulatory visits among children $<5$ years. We expect lower rates of rotavirus after introduction of the vaccine in early 2010, which will increase the relative importance of norovirus as an etiology of severe diarrheal disease in children [14]. This is consistent with other reports showing high levels of norovirus I sporadic cases as well as outbreaks in Guatemala $[9,14,15]$. Among those $\geq 1$ years old, bacterial pathogens, particularly diarrheagenic E. coli, played a more prominent role. In our surveillance, $18 \%$ of ambulatory patients in this age group had diarrheagenic E. coli, as did 10\% of those hospitalized. In addition, Shigella was detected in 6\% and Campylobacter was detected in $3 \%$ overall, but Salmonella detection

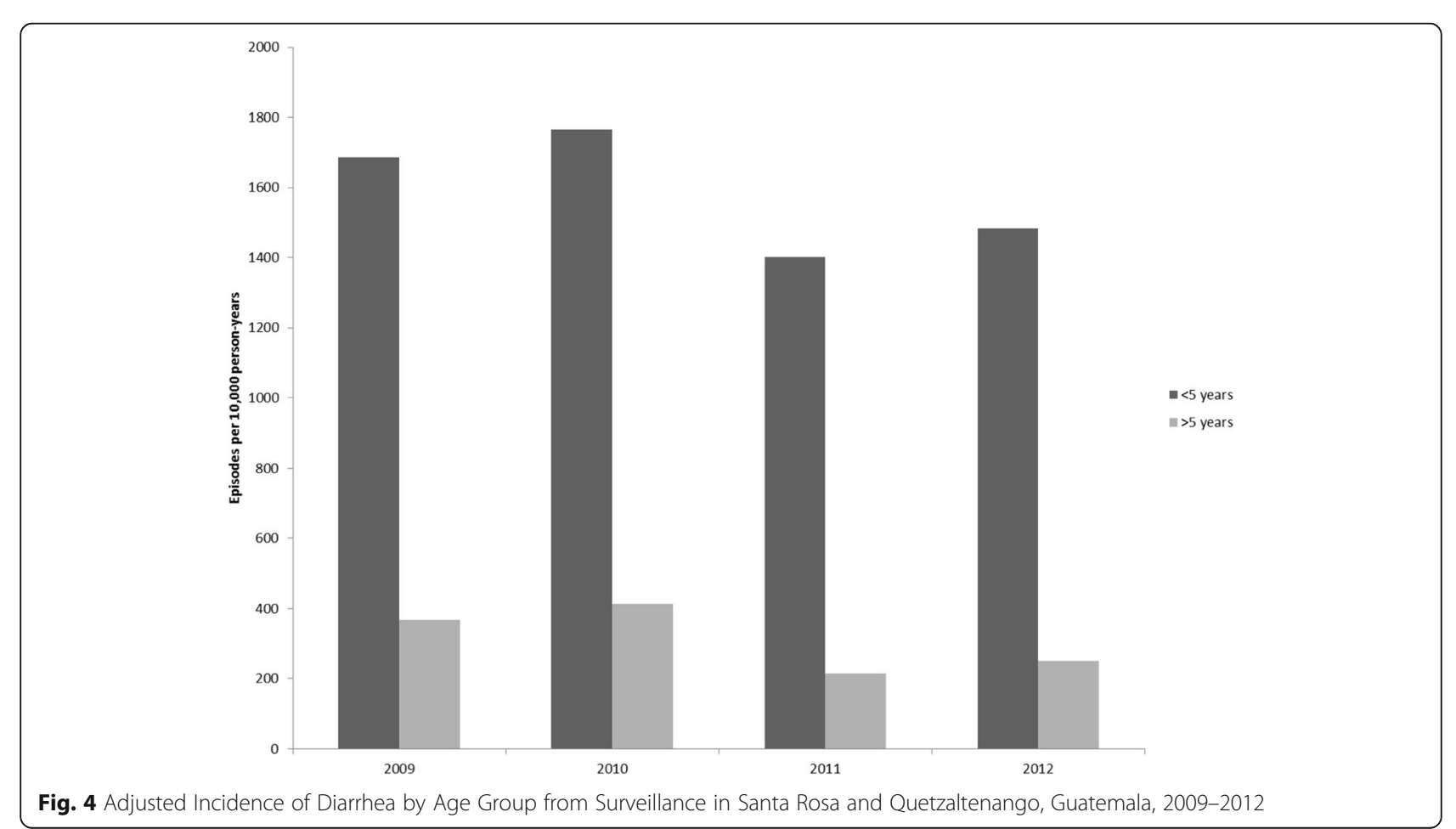


Table 2 Etiology of diarrhea by age group and sex from laboratory-based disease surveillance conducted from July 2008 to July 2009, Santa Rosa and Quetzaltenango, Guatemala

\begin{tabular}{|c|c|c|c|c|c|c|c|c|c|c|c|}
\hline \multirow{3}{*}{$\begin{array}{l}\text { Age } \\
\text { Group } \\
\text { (Years) } \\
<1\end{array}$} & \multirow{3}{*}{$\begin{array}{l}\text { Specimens } \\
\text { Tested } \\
294\end{array}$} & \multicolumn{10}{|c|}{ Etiologic Category* } \\
\hline & & \multicolumn{2}{|l|}{$\overline{\text { Viral }}$} & \multicolumn{2}{|c|}{ Bacterial } & \multicolumn{2}{|c|}{ Parasitic } & \multicolumn{2}{|c|}{ Multiple } & \multicolumn{2}{|c|}{ Unknown } \\
\hline & & 97 & $33 \%$ & 47 & $16 \%$ & 3 & $1 \%$ & 29 & $10 \%$ & 118 & $40 \%$ \\
\hline $1-4$ & 533 & 99 & $19 \%$ & 124 & $23 \%$ & 19 & $4 \%$ & 34 & $6 \%$ & 257 & $48 \%$ \\
\hline $5-19$ & 254 & 15 & $6 \%$ & 48 & $19 \%$ & 23 & $9 \%$ & 15 & $6 \%$ & 153 & $60 \%$ \\
\hline $20-49$ & 202 & 12 & $6 \%$ & 34 & $17 \%$ & 3 & $2 \%$ & 7 & $4 \%$ & 146 & $72 \%$ \\
\hline$>50$ & 98 & 8 & $8 \%$ & 21 & $21 \%$ & 1 & $1 \%$ & 1 & $1 \%$ & 67 & $68 \%$ \\
\hline Total & 1381 & 235 & $17 \%$ & 275 & $20 \%$ & 50 & $4 \%$ & 86 & $6 \%$ & 741 & $54 \%$ \\
\hline
\end{tabular}

*Etiologic category assigned based on detection of pathogens within a given category (e.g., viral, bacterial, and parasitic) and in the absence of pathogens from other categories

was lower than expected at $1 \%$ [16]. Likewise, Cryptosporidium detection was lower than in the Global Enteric Multicenter Study (GEMS) [13]. Optimal testing for $E$. histolytica was not conducted, but entamoeba cysts were seldom observed.

We also found from the health utilization surveys that although the majority of patients sought care for their illness outside the home, less than $25 \%$ went to a government health facility. Even though children $<5$ years old most often sought care at a government health facility, only 51\% of these children sought care at these facilities. Low utilization of government-run services for diarrhea are consistent with those of another study in a similar population in Guatemala $[8,17,18]$. In Guatemala, government facilities are responsible for reporting diarrheal diseases, and national surveillance systems such as the Rotavirus Sentinel Surveillance, located is facility-based, as in many other countries [19-21]. Our findings show the national rotavirus sentinel surveillance in Guatemala may be capturing about half of cases of diarrhea among children $<5$ years of age.
While these surveys conducted in two of Guatemala's 22 administrative areas may not be representative, attempts should be made to correct local and national surveillance data intended for describing incidence and hospitalization rate estimates adjusting for healthcare seeking practices.

Our study is subject to several limitations. First, we did not include control groups for comparison of etiologic detection rates. Results from GEMS showed a high pathogen detection, particularly norovirus, Campylobacter, and Giardia in asymptomatic control children, suggesting that our data may have over-represented their importance as pathogens [13]. Second, we found much lower incidences than in other reports. A recent systematic review reported a median global incidence for diarrheal in children < 5 years of age at 3.4 episodes per person-year. The incidence in Guatemala in this systematic review was 4.3 per child year [1]. Another systematic review among older children and adults also reported a higher incidence rate than ours, but lacked data from Central and South America [4, 22]. There are several

Table 3 Detection of Specific Pathogens among Diarrhea cases by Healthcare Facility Type and Age Group in Santa Rosa and Quetzaltenango, Guatemala

\begin{tabular}{|c|c|c|c|c|c|c|c|c|c|c|c|c|}
\hline \multirow[t]{3}{*}{ Pathogen Detected* } & \multicolumn{6}{|c|}{ Ambulatory } & \multicolumn{6}{|c|}{ Hospital } \\
\hline & \multicolumn{2}{|c|}{$<5$ years } & \multicolumn{2}{|c|}{$\geq 5$ years } & \multicolumn{2}{|c|}{ All ages } & \multicolumn{2}{|c|}{$<5$ years } & \multicolumn{2}{|c|}{$\geq 5$ years } & \multicolumn{2}{|c|}{ All ages } \\
\hline & \multicolumn{2}{|c|}{$n=630(\%)$} & \multicolumn{2}{|c|}{$n=507(\%)$} & \multicolumn{2}{|c|}{$n=1137(\%)$} & \multicolumn{2}{|c|}{$n=216(\%)$} & \multicolumn{2}{|c|}{$\overline{n=48(\%)}$} & \multicolumn{2}{|c|}{$n=264(\%)$} \\
\hline \multicolumn{13}{|l|}{ Bacteria } \\
\hline Diarrheagenic E. coli & 128 & $21 \%$ & 89 & $18 \%$ & 217 & $19 \%$ & 33 & $16 \%$ & 5 & $10 \%$ & 38 & $15 \%$ \\
\hline Shigella & 35 & $6 \%$ & 28 & $6 \%$ & 63 & $6 \%$ & 6 & $3 \%$ & 3 & $6 \%$ & 9 & $4 \%$ \\
\hline Campylobacter & 30 & $5 \%$ & 5 & $1 \%$ & 35 & $3 \%$ & 7 & $3 \%$ & 0 & - & 7 & $3 \%$ \\
\hline Salmonella & 1 & $<1 \%$ & 3 & $1 \%$ & 4 & $<1 \%$ & 1 & $1 \%$ & 1 & $2 \%$ & 2 & $1 \%$ \\
\hline \multicolumn{13}{|l|}{ Virus } \\
\hline Norovirus & 104 & $17 \%$ & 30 & $6 \%$ & 134 & $12 \%$ & 61 & $30 \%$ & 4 & $8 \%$ & 65 & $26 \%$ \\
\hline Rotavirus & 50 & $8 \%$ & 19 & $4 \%$ & 69 & $6 \%$ & 49 & $24 \%$ & 0 & $==$ & 49 & $19 \%$ \\
\hline \multicolumn{13}{|l|}{ Protozoa } \\
\hline Giardia & 27 & $4 \%$ & 34 & $7 \%$ & 61 & $5 \%$ & 2 & $1 \%$ & 0 & - & 2 & $1 \%$ \\
\hline Cryptosporidium & 6 & $1 \%$ & 1 & $<1 \%$ & 7 & $1 \%$ & 2 & $1 \%$ & 0 & - & 2 & $1 \%$ \\
\hline
\end{tabular}

*Multiple pathogens may be detected in a given specimen; thus, some diarrhea cases are counted in more than one row 
reasons why the incidence for diarrhea in these systematic reviews was higher than in our study. We used healthcare facility data and extrapolated our findings based on healthcare seeking data from household surveys, and might have overestimated healthcare seeking rates, which would lead to a lower extrapolated incidence rate. During the household surveys, we ascertained healthcare seeking practices for the 30 days prior to interview, and recall bias may have led to an underestimation. The household survey in Santa Rosa was conducted during the rotavirus season, which could have also led to an overestimation of healthcare seeking practices, resulting in lower adjusted incidence rates. Finally, we may have under-ascertained diarrhea cases presenting at surveillance facilities. In our study patients presenting to the emergency department, but not admitted to the hospital, were not eligible for inclusion. Only patients who were hospitalized were enrolled in the surveillance system, as one of the main objectives for the implementation of the surveillance system was to capture the more severe diarrhea cases. Since many patients with diarrhea receive hydration in the emergency department and are then discharged home, this may have led to much lower numbers reported as compared to other studies. Despite these limitations, this surveillance system yielded high quality results with laboratory-based detection of enteric pathogens, through consistent surveillance with dedicated nurses and laboratorians, and the use of PDAs and electronic transfer of data.

\section{Conclusions}

We estimated that in our surveillance sites in Guatemala, approximately 1 in 15 persons suffers and seeks medical care for a diarrheal disease episode each year. Our estimates were based from active laboratory-based diarrhea surveillance among ambulatory and hospitalized patients, and observed rates were adjusted with data from surveys describing healthcare seeking practices. Pathogen specific data highlighted the importance of viruses and diarrheagenic $E$. coli as an important etiology for diarrhea in this region. Shigella remains an important pathogen with considerations for increase antimicrobial resistance. This investigation highlights the importance of strengthening laboratory capacity in Guatemala for rapid detection and control of health threats, and the evaluation of public health interventions to inform adequate control and prevention strategies that could reduce the burden of diarrheal diseases.

\section{Disclaimer}

Reported findings and conclusions are those of the authors and do not necessarily represent the official position of the CDC.

\section{Supplementary information}

Supplementary information accompanies this paper at https://doi.org/10. 1186/s12889-019-7720-2.

Additional file 1. Household Survey. Questionnaire used for the household survey.

Additional file 2. Surveillance Questions. Questions used for patients enrolled in the surveillance system.

\section{Abbreviations}

CDC: US Centers for Disease Control and Prevention; EIA: Enzyme Immunoassay; GPS: Global Positioning System; PDAs: Personal Digital Assistant Devices; RT-qPCR: Reverse Transcription quantitative polymerase chain reaction; SAS: Statistical Analysis System; UVG: Universidad del Valle de Guatemala

\section{Acknowledgments}

We acknowledge the participation of the families in Santa Rosa and Quetzaltenango. We also thank all the physicians, nurses, and epidemiologists at ministry of health. This work would not have been possible without the technical contributions of numerous epidemiologists, scientists, laboratorians, data programmers, and field support staff from the UVG and CDC. We specifically like to thank Laura Grajeda for her support developing the maps presented in this manuscript.

\section{Authors' contributions}

All co-authors have read and approved the current version of this manuscript and approved it for publication. Below the specific contributions from each co-author: WA: conception and design of the household surveys and surveillance system; acquisition, analysis, and interpretation of data for the household survey and the surveillance system; lead writer for the drafted the work. AJH: Acquisition, analysis, and interpretation of data for the household survey and the surveillance system; substantively revised draft AND to have approved the submitted version. $\mathrm{OH}$ : Conception and design of the household surveys and surveillance system; acquisition, analysis, and interpretation of data for the household survey and the surveillance system; substantively revised draft and have approved the submitted version. BL: Conception and design of the household surveys and surveillance system; acquisition, analysis, and interpretation of laboratory data for the surveillance system; substantively revised draft and have approved the submitted version. CB: Acquisition of data for the surveillance system; substantively revised draft and have approved the submitted version. JCM: Conception and design of the household survey and surveillance system in Quetzaltenango; acquisition and interpretation of data for the surveillance system; substantively revised draft and have approved the submitted version. LR: Conception and design of the household survey and surveillance system in Santa Rosa; acquisition and interpretation of data for the surveillance system; substantively revised draft and have approved the submitted version. SPM: Conception and design of the household surveys and surveillance system; interpretation of data for the household survey and the surveillance system; substantively revised draft and have approved the submitted version. OM: Conception and design of the household survey in Quetzaltenango; acquisition and interpretation of data for the household survey; substantively revised draft and have approved the submitted version. AE: Conception and design of the surveillance system; acquisition, analysis, and interpretation of laboratory data for the surveillance system; substantively revised draft and have approved the submitted version. MRL: Conception and design of the surveillance system; acquisition, analysis, and interpretation of laboratory data for the surveillance system; substantively revised draft and have approved the submitted version. MBP: Conception and design of the surveillance system; acquisition, analysis, and interpretation of laboratory data for the surveillance system; substantively revised draft and have approved the submitted version. GG: Acquisition, analysis, and interpretation of laboratory data for the surveillance system; substantively revised draft and have approved the submitted version. JV: Acquisition, analysis, and interpretation of laboratory data for the surveillance system; substantively revised draft and have approved the submitted version. NG: Acquisition, analysis, and interpretation of laboratory data for the surveillance system; substantively revised draft and have approved the submitted version. UP: Conception and design of the surveillance system; interpretation of data for the surveillance system; substantively revised draft and have 
approved the submitted version. EDM: Conception and design of the household surveys and surveillance system; interpretation of data for the household survey and the surveillance system; substantively revised draft and have approved the submitted version. JPM: Conception and design of the surveillance system; interpretation of data for the surveillance system; substantively revised draft and have approved the submitted version. JPB: Conception and design of the surveillance system; interpretation of data for the surveillance system; substantively revised draft and have approved the submitted version. KAL: Conception and design of the household surveys and surveillance system; acquisition, analysis, and interpretation of data for the household survey and the surveillance system; substantively revised draft and have approved the submitted version.

\section{Funding}

Supported in part by Cooperative Agreement UO1 GH000028-02 from the Centers for Disease Control and Prevention. CDC employees provided direct technical assistance to the ministry and university officials, and were directly engaged in all aspects of the household surveys and the surveillance system.

\section{Availability of data and materials}

Regarding data availability, all data are kept in secured servers at the UVG given they contain personally identifiable and sensitive clinical information protected by the Health Insurance Portability and Accounting Act (HIPAA). Redacted deidentified versions of the data are available upon request from the corresponding author.

\section{Ethics approval and consent to participate}

Protocols for both the household surveys and the surveillance system received approval from the institutional review boards of the US Centers for Disease Control and Prevention (Atlanta, GA; CDC IRB protocol \#5150), and of the Universidad del Valle de Guatemala (Guatemala City, Guatemala). In addition, the protocol was reviewed and received approval from the Guatemalan Ministry of Public Health and Welfare (Guatemala City, Guatemala). The protocols included institutional review board approval to enroll minors $<17$ years of age with consent from the parent or caregiver, and assent if the child was 7 years of age or older.

For the household surveys, heads of household $\geq 18$ years old were asked for written, informed consent for them and the members of the household to participate in the survey. Head of household was defined as the primary caregiver of most children in household or the oldest person $>18$ years old in the household. All other members of the household $\geq 18$ years old were also asked to provide verbal consent, and children aged 7 through 17 years provided verbal assent, as approved by institutional review boards. Verbal consent was approved as there was no risk for the household members to participate in a small subset of question from the survey, and heads of households had provided written informed consent.

For the surveillance system, patients $\geq 18$ years old and caregivers of children $<18$ years old had to provide written, informed consent in order for them or their children to participate in the surveillance. For children aged 7 through 17 years, written assent was required in addition the caregivers written consent. For children younger than seven years we only obtained written consent to participate from their caregivers, as approved by the institutional review boards. Trained nurses informed participants about the study, including that their participation was voluntary and confidential. An informational sheet with a description of the study and contact information for the investigators was also provided. Signed consent forms were delivered to the investigators at the UVG daily.

\section{Consent for publication}

Not applicable.

\section{Competing interests}

The authors declare that they have no competing interests

\section{Author details}

${ }^{1}$ Center for Global Health, US Centers for Disease Control and Prevention, 1600 Clifton Road, NE, Mailstop V24-5, Atlanta, GA 30333, USA. 'National Center for Immunization and Respiratory Diseases, CDC, Atlanta, USA. ${ }^{3}$ National Center for Emerging and Zoonotic Infectious Diseases, CDC, Atlanta, USA. ${ }^{4}$ Centro de Estudios en Salud, Universidad del Valle de
Guatemala, Guatemala City, Guatemala. ${ }^{5}$ Ministry of Public Health and Welfare, Guatemala City, Guatemala.

\section{Received: 6 May 2019 Accepted: 9 October 2019}

Published online: 22 October 2019

\section{References}

1. Black RE, et al. Global, regional, and national causes of child mortality in 2008: a systematic analysis. Lancet. 2010;375(9730):1969-87.

2. Bresee JS, et al. The etiology of severe acute gastroenteritis among adults visiting emergency departments in the United States. J Infect Dis. 2012; 205(9):1374-81.

3. Liu L, et al. Global, regional, and national causes of child mortality in 2000 13, with projections to inform post-2015 priorities: an updated systematic analysis. Lancet. 2015;385(9966):430-40.

4. Fischer Walker $\mathrm{CL}$, et al. Diarrhea incidence in low- and middle-income countries in 1990 and 2010: a systematic review. BMC Public Health. 2012;12:220.

5. Parashar UD, Bresee JS, Glass RI. The global burden of diarrhoeal disease in children. Bull World Health Organ. 2003;81(4):236.

6. Walker $\mathrm{CL}$, Black RE. Diarrhoea morbidity and mortality in older children, adolescents, and adults. Epidemiol Infect. 2010;138(9):1215-26.

7. Cortes J, et al. Rotavirus disease burden among children $<5$ years of age--Santa Rosa, Guatemala, 2007-2009. Tropical Med Int Health. 2012;17(2):254-9.

8. Delgado E, Sorensen SC, Van der Stuyft P. Health seeking behaviour and self-treatment for common childhood symptoms in rural Guatemala. Ann Soc Belg Med Trop. 1994;74(2):161-8.

9. Arvelo W, et al. Diagnostic performance of rectal swab versus bulk stool specimens for the detection of rotavirus and norovirus: implications for outbreak investigations. J Clin Virol. 2013;58(4):678-82.

10. Estevez $\mathrm{A}$, et al. Prevalence and genetic diversity of norovirus among patients with acute diarrhea in Guatemala. J Med Virol. 2013;85(7):1293-8.

11. Velasquez DE, et al. Short report: molecular insights for Giardia, Cryptosporidium, and soil-transmitted helminths from a facility-based surveillance system in Guatemala. Am J Trop Med Hyg. 2011;85(6):1141-3.

12. Estevez $\mathrm{A}$, et al. Prevalence and genetic diversity of norovirus among patients with acute diarrhea in Guatemala. J Med Virol. 2013;85(7):1293-8.

13. Kotloff $\mathrm{KL}$, et al. Burden and aetiology of diarrhoeal disease in infants and young children in developing countries (the global enteric multicenter study, GEMS): a prospective, case-control study. Lancet. 2013;382(9888):209-22.

14. Diez-Valcarce $M$, et al. Prevalence and genetic diversity of viral gastroenteritis viruses in children younger than 5 years of age in Guatemala, 2014-2015. J Clin Virol. 2019;114:6-11.

15. Arvelo W, et al. Norovirus outbreak of probable waterborne transmission with high attack rate in a Guatemalan resort. J Clin Virol. 2012;55(1):8-11.

16. Walker $\mathrm{CL}$, et al. Global burden of childhood pneumonia and diarrhoea. Lancet. 2013;381(9875):1405-16.

17. Schooley J, et al. Factors influencing health care-seeking behaviours among Mayan women in Guatemala. Midwifery. 2009;25(4):411-21.

18. van der Stuyft P, Delgado E, Sorensen SC. Utilisation rates and expenditure for public and private, curative-care services in semi-urban Guatemala. Ann Trop Med Parasitol. 1997;91(2):209-16.

19. de Oliveira $L H$, et al. Sentinel hospital surveillance for rotavirus in latin american and Caribbean countries. J Infect Dis. 2009;200(Suppl 1):S131-9.

20. Guardado JA, et al. Rotavirus in El Salvador: an outbreak, surveillance and estimates of disease burden, 2000-2002. Pediatr Infect Dis J. 2004;23(10 Suppl):S156-60.

21. Munford V, et al. Rotavirus gastroenteritis in children in 4 regions in Brazil: a hospital-based surveillance study. J Infect Dis. 2009;200(Suppl 1):S106-13.

22. Flint $J A$, et al. Estimating the burden of acute gastroenteritis, foodborne disease, and pathogens commonly transmitted by food: an international review. Clin Infect Dis. 2005;41(5):698-704.

\section{Publisher's Note}

Springer Nature remains neutral with regard to jurisdictional claims in published maps and institutional affiliations. 